\begin{tabular}{lll} 
Pădagogik Aktuelle Forschungs- und Medienprojekte \\
\cline { 2 - 4 }
\end{tabular}

\section{Explorative Untersuchung der Nachhaltigkeit innovativer medienpädagogischer Projek- te für und mit Seniorinnen und Senioren ${ }^{1}$}

\section{KATHARINA GMEINWIESER}

Digitale Medien durchdringen die Handlungsräume der gegenwärtigen Gesellschaft. Smartphones und Apps, Social Media und Tablet-PCs beherrschen die Kommunikationswege. Ältere Menschen wurden nicht in diese Medienwelt hineingeboren. Sie müssen sich aktiv darum bemühen, den Anschluss an die Mediengesellschaft nicht zu verlieren. Viele jedoch konnten in ihrem Berufsleben bereits notwendige Grundkenntnisse erwerben und treten nicht als Unwissende in das Seniorenalter ein. Vorrangig wurden diese neuen Medien dabei als Arbeitsgeräte genutzt und verstanden. Wenn sich das Berufsleben dann dem Ende zuneigt, kann und sollte sich dieses Verständnis dahingehend ändern, dass neue Medien auch als Bereicherung des Alltags entdeckt werden.

Seniorinnen und Senioren ${ }^{2}$ stehen immer häufiger im Zentrum medienpädagogischer Bildungsangebote. Das Weiterbildungsangebot in den Volkshochschulen und anderen Einrichtungen der Erwachsenen- und Seniorenbildung wächst in diesem Bereich langsam an, denn die Nachfrage besteht. Doch innovative Projekte, die von der Vermittlung von (Computer-)Grundkenntnissen abweichen, sind bisher noch selten.

\section{Interesse an medienpädagogi-} scher Seniorenbildung wächst

Wie die Vorausberechnungen des Statistischen Bundesamtes (vgl. Statistisches Bundesamt 2009) in Bezug auf die Entwicklung der Bevölkerung in Deutschland zeigen, wächst die Gruppe der über 60-Jährigen bis zum Jahr 2060 erheblich an. Ein wesentlicher Faktor der gestiegenen Lebenserwartung ist die gesteigerte Gesundheit älterer Menschen (vgl. Berner/ Tesch-Römer/ Wurm 2013, S. 4), welche sich zudem durch „einen höheren Bildungsgrad, vielfältige Kompetenzen und einer zunehmenden Be- reitschaft zum Engagement aus[zeichnen; K.G.]. Viele wollen auch im Alter lernen und schrecken vor anspruchsvollen Inhalten nicht zurück“ (de Groote/ Nebauer 2008, S. 189).

Ältere Menschen werden als Zielgruppe kultureller und medienpädagogischer (Weiter-)Bildungsangebote also immer relevanter. „Daraus ist $\mathrm{zu}$ folgern: Lebenslanges Lernen ist $[. .$.$] zu einer Existenznotwendig-$ keit geworden. Es gewährleistet dem Individuum soziale Teilhabe und psychophysisches Wohlbefinden“ (Kolland 2008, S. 174). Bisher gibt es aber nur wenige medienpädagogische Bereiche, die sich dieses Wandels annehmen. Die Angebote für Senioren konzentrieren sich meist auf das Entgegenwirken von Defiziten im Umgang mit neuen Medien. Ästhetisch-gestalterische Vermittlung findet nur selten statt. Die Konzentration liegt darauf, „Lücken zu füllen, die entstanden sind durch einerseits die beschleunigte Medienentwicklung und andererseits das mentale und praktische Hinterherhinken der älteren Menschen“ (Schorb 2011, S. 63). Medien stellen für diese Menschen ebenso wie für Kinder und Jugendliche eine Form des persönlichen Ausdrucks und der Kommunikation dar und sollten deshalb von allen Altersgruppen gleichermaßen genutzt werden können (vgl. Schorb 2011, S. 56). Mit der Teilnahme an Kursen und Projekten geht es „[d]en Älteren [...] um die Kulturteilhabe, um die Suche nach neuen Rollen, wenn berufliche oder familiäre Verpflichtungen wegfallen, sowie um allgemeine persönlichkeitsbildende Ziele" (de Groote/ Fricke 2010, S. 22). Hierzu gehört auf der einen Seite, den Anschluss an die moderne technisierte Welt nicht zu verlieren, auf der anderen Seite steht die Aneignung künstlerischer Kompetenzen. Hierfür müssen Überlegungen angestellt und Konzepte entworfen werden, die nachvollziehbare Hilfestellungen für die medienpädagogische Praxis mit Senioren bieten.

Ein breites Forschungsfeld eröffnet sich

Im Bereich der Forschung ist dieses Feld noch unzureichend erschlossen. „Überblickt 
man den Erkenntnisstand medienwissenschaftlicher und speziell medienpädagogischer Forschung, so finden sich nur wenige tiefer gehende Befunde zur Bedeutung der Medien im höheren Erwachsenenalter" (Hartung/ Reißmann/ Schorb 2005, S. 124). Empirische Studien lassen zwar auf Interessen und Nutzungsverhalten der wachsenden Altersgruppe Senioren schließen (vgl. ARD/ ZDF-Onlinestudie 2013, BITCOM e.V. 2013), offen bleibt jedoch, welche Bedingungen zu dieser Nutzung führen, welche Bedeutung die entsprechende Nutzung für die Zielgruppe innehat oder wie Nutzungsvorlieben älterer Menschen entstehen. Hier öffnet sich ein breites Forschungsfeld.

Bisher existieren kaum Leitlinien zum didaktischen und methodischen Aufbau medienpädagogischer Projektangebote für diese Zielgruppe. Die Veröffentlichung „Kulturkompetenz 50+“ (de Groote/ Fricke 2010) bietet hierfür, als Ratgeber für die Kulturarbeit mit Senioren, einen Anfang. Ein wesentlicher Teil des Werkes bietet Praxistipps für die Planung sowie die Durchführung von Kulturangeboten für Ältere (vgl. de Groote/Fricke 2010, S. 27ff., S. 45ff.). Die Ausarbeitung von de Groote/ Fricke stellt eine Basis dar, auf der die medienpädagogische Praxis aufbaut. Die Nachhaltigkeit der Projektangebote ist jedoch häufig nicht gesichert. „Wenn Projekte enden, bröckeln meist die mühsam aufgebauten Strukturen." (de Groote/ Nebauer 2008, S. 23). Unter Nachhaltigkeit wird hier die Etablierung eines zeitlich begrenzten Projektangebots als dauerhafte Einrichtung verstanden. Diese Auffassung von Nachhaltigkeit orientiert sich stärker an der Zukunft des Projektes selbst sowie an der Etablierung des Projektkonzepts und weniger an der nachhaltigen Lernwirkung bei den Teilnehmenden. Diesem Aspekt widmet sich die hier beschriebene Forschung.

\section{Nachhaltige Bildungsansprüche}

„Damit Lernen nachhaltig wird, braucht es nicht das Vermitteln und Abprüfen von Faktenwissen, es braucht das Verstehen vom Lernen selbst (Selbstkompetenz)“ (Ladner 2013, S. 23). Dies ist insbesondere in Bezug auf den raschen Entwicklungsfortschritt in der Gesellschaft bedeutsam, „weil es [...] aufgrund der schnellen Veralterungsrate einmal erworbenen Wissens und struktureller Unsicherheiten der Lebensführung wichtig ist, dass Menschen sich Neues schnell aneignen können und auch in veränderten Situationen handlungsfähig bleiben" (Schüßler 2007, S. 96). Die Ausbildung von Kompetenzen, darunter auch die Medienkompetenz (vgl. Bogen u.a. 2005, S. 12), bildet die Basis lebenslangen Lernens und stellt das Ziel nachhaltigen Lernens dar.

Schüßler definiert eine temporale, eine strukturelle und eine graduelle Dimension nachhaltigen Lernens, welche noch weiter ausdifferenziert werden und in Wechselwirkung zueinander stehen. Diese Unterteilung bietet die Möglichkeit der „Untersuchung nachhaltiger Lernprozesse“ (Schüßler 2007, S. 212). Auf der temporalen Ebene bezeichnet nachhaltiges Lernen einen Prozess, für welchen Zeit bereitgestellt wird, um ,mit Abstand auf aktuelle Handlungsproblematiken zu schauen und über diese zu reflektieren“ (Schüßler 2007, S. 208). Die zweite Dimension nachhaltigen Lernens bezieht sich auf die Struktur und unterteilt sich in eine Kontext-, eine Subjektund eine Wissenssubdimension. Der Kontext bezieht sich auf den lernkulturellen Rahmen der Bildungssituation. Er „steht in Wechselwirkung zum Subjekt. Habitus, Lernbiographie, Motivationen, Interessen sowie Rollenerwartungen entwickeln sich durch Enkulturation in eine bestimmte (Lern-)Kultur" (Schüßler 2007, S. 208). Für die Entwicklung von Wissen und die Ausbildung von Kompetenzen ist es wichtig, dass die Lernenden ihren persönlichen Nutzen in den Informationen erkennen können, „wenn also das Lernangebot [...] als für [ihre; K.G.] Zwecke und Handlungsprobleme relevant wahrgenommen wird und somit auf konkrete Situationen bezogen werden kann" (Schüßler 2007, S. 210). An dritter Stelle steht die graduelle Dimension nachhaltigen Lernens, welche in Tiefe, Kohärenz und Breite ausdifferenziert wird. „Die Tiefendimension fokussiert hier den Grad der Irritation, Betroffenheit und emotionalen Resonanz", während sich die Kohärenz ,auf den Grad der Anschlussfähigkeit des Gelernten an bisheriges Wissen und die Sicherung einer biographischen Kontinuität" bezieht (Schüßler 2007, S. 210). Die Breite bezieht sich auf den Radius der Einsetzbarkeit des neu erworbenen Wissens. „Im günstigsten Falle drückt sich der Grad der Breite darin aus, dass das Gelernte auch in anderen Kontexten zur Anwendung kommt und schließlich intuitiv sowie situationsangemessen eingesetzt wird“ (Schüßler 2007, S. 211).

\section{Innovative Projekte bilden die Grundlage zur Erforschung von Nachhaltigkeit}

Aus einem Angebot von vier Projekten, welche sich durch ihr innovatives Design vom herkömmlichen medienpädagogischen Angebot für Ältere abgrenzten, wurden Personen als Interviewpartner ausgewählt. Sie sollten in die Konzeption und/oder Durchführung der jeweiligen Projekte involviert (gewesen) sein. 
Die Personen sollten selbst bereits im medienpädagogischen Kontext mit Senioren gearbeitet haben, um aus dieser Erfahrung heraus als Experten im Interview zu fungieren. Ziel der Interviews war es herauszufinden, wie innovative medienpädagogische Projektangebote gestaltet werden müssen, um nachhaltige Lernprozesse bei älteren Teilnehmenden anzuregen.

Bei den Projekten handelte es sich um eine beispielhafte Auswahl von Angeboten, die innerhalb der letzten Jahre im Raum Stuttgart und in Württemberg durchgeführt wurden. In zwei Fällen wurden die Projekte durch bundesweite Träger in dieser Region angeboten. Es handelt sich somit um eine explorative Untersuchung. Die Auswahlparameter waren dabei die Zielgruppe Senioren und die medienpädagogische Schwerpunktsetzung der Projekte. Intergenerationelle Projekte wurden nicht berücksichtigt. Ein weiteres charakteristisches Merkmal von Projekten ist die Zeit. ,Jedes Projekt ist [...] zeitlich befristet, hat einen Anfang und ein Ende“ (Reichert-Garschhammer 2013, S. 19). Die Erstellung eines Produkts zum Abschluss eines Projektes wird dem Projektprozess unterstellt (vgl. Gudjons 2008, S. 88). Eigenständiges Handeln wird hierbei als „unverzichtbare Form für den Erwerb und die Entwicklung von Wissen und Kompetenzen" (Pfäffli 2005, S. 189) angesehen und zählte zu den Auswahlkriterien der beispielhaften Projekte.

Bei der Untersuchung innovativer Projekte musste geklärt werden, was unter dem Begriff in diesem Kontext verstanden wird. Innovation kann sich sowohl auf einen Gegenstand als auch auf die Art des Angebots oder den Prozess beziehen (vgl. Fischer/ Nemnich 2013, S. 170). So besitzt jedes der ausgewählten Projekte einen eigenen innovativen Charakter.

Das Projekt ,Silberwissen', welches von Wikimedia-Deutschland im Rahmen des Forschungsprojekts TAO der Europäischen Union ins Leben gerufen und von 2010 bis 2013 in Zusammenarbeit mit dem ZAWIW in Ulm durchgeführt wurde, ermöglichte es den Teilnehmenden selbst als Autoren für die Online-Enzyklopädie, Wikipedia' aktiv zu werden (vgl. ZAWiW 2013). Das Projekt erwies sich in dem Sinne als innovativ, da es vorrangig darauf abzielte, den Teilnehmenden eine Plattform zu bieten, Wissen zu sammeln und Artikel zu veröffentlichen. Hierzu wurde ein betreuter Einstieg geboten, der den Teilnehmenden im weiteren die Möglichkeit des eigenständigen wissenschaftlichen Ausdrucks und eine Öffentlichkeit bot.
Der innovative Aspekt des zweiten ausgewählten Projekts ,Smartphone4BestAger", eine Projektinitiative der Volkshochschulen Baden-Württemberg, zeichnet sich in der Wahl des Mediums ab. Senioren lernen in Einsteigerkursen, welche Art von Endgeräten es gibt und welche Vorteile diese für den Alltag bieten. In weiterführenden Kursen werden dann spezielle Funktionen konkret anhand eines bestimmten Betriebssystems veranschaulicht und können direkt mit den eigenen Geräten erprobt werden (vgl. Volkshochschulverband Baden-Württemberg). Diese Nachfolgekurse verfolgen einen stark handlungsorientierten Ansatz. Durch das Ausprobieren mit dem eigenen Gerät ist es nicht mehr notwendig, das neu erworbene Wissen auf das eigene Smartphone oder auch einen Tablet-PC zu transferieren.

Das Projekt ,Tablet-PCs für Seniorinnen und Senioren' wird seit dem Jahr 2012 in Kooperation mit dem Mobilfunkanbieter e-plus und dessen Marke BASE durchgeführt. Hierbei werden Tablet-PCs, ausgestattet mit einer Internetflatrate, für den Zeitraum von vier Wochen an Senioreneinrichtungen ausgeliehen. 20 Prozent der Geräte werden der Einrichtung nach dem Ausleihzeitraum dauerhaft überlassen; die Internetflatrate wird dann für zwölf Monate bereitgestellt. Die Nutzung von Tablet-PCs mit Touchscreen zur Heranführung von älteren Menschen an das Internet stellt eine Innovation in diesem Bereich dar. Die Senioren haben die Möglichkeit, unter Anleitung die Geräte kennenzulernen und auszuprobieren, ohne dabei eine vertragliche Verpflichtung einzugehen. Somit wird ein niedrigschwelliger, begleiteter Einstieg in die Nutzung des neuen Mediums ermöglicht (vgl. Stiftung Digitale Chancen).

Bei dem Projekt ,Senioren-Internet-Helfer ${ }^{6}$ des Netzwerks für Senioren-Internet-Initiativen Baden-Württemberg erlernen Senioren die notwendigen Grundlagen in der Arbeit mit neuen Medien und können diese anschließend an Personen ihrer Altersgruppe weitervermitteln. Unterstützt wird das Netzwerk der Senioren-Internet-Initiativen Baden-Württemberg dabei vom ZAWiW in Ulm (vgl. Netzwerk für Senioren-InternetInitiativen Baden-Württemberg). Die Peerto-peer-Vermittlung der Senioren stellt dieses Projekt als besonders innovativ heraus. $\mathrm{Zu}-$ dem geht die Kompetenzvermittlung über die Nutzung digitaler Medien hinaus und bietet eine Hilfestellung bei der Etablierung von Medienangeboten in Gemeinden.

\section{Methodisches Vorgehen}

Der Erstkontakt mit den Interviewpartnern wurde vorrangig per E-Mail hergestellt, in 
einem Fall auch telefonisch. Bei der Vereinbarung der Interviewtermine wurde dafür Sorge getragen, dass der Aufwand für die Interviewpartner möglichst gering gehalten wurde, weshalb die Orte von den Interviewpartnern ausgewählt wurden. Zwei der vier Interviews wurden aufgrund der räumlichen Distanz telefonisch durchgeführt. In einem Fall waren zwei Projektverantwortliche während des Interviews anwesend, wobei eine Person die Funktion des Hauptinterviewpartners übernahm und die zweite nur ergänzend eingreifen konnte.

Als Erhebungsinstrument diente das nichtstandardisierte, leitfragengestützte Experteninterview. Hierbei standen zunächst die praktischen Erfahrungen und Projekte der Interviewpartner im Vordergrund. Die Fragen, die sich daran anschlossen, bezogen sich stärker auf den Begriff des nachhaltigen Lernens, dann auf persönliche Praxiserfahrungen diesbezüglich. Die Interviewpartner wiesen unterschiedliche Professionalisierungsgrade in Bezug auf die Durchführung medienpädagogischer Bildungsprojekte und -angebote auf. So zählten zu den Befragten sowohl Ehrenamtliche als auch Geschäftsführende und Personen, die selbst zur Altersgruppe der Senioren zählten. Es zeigte sich, dass durch die Expertise dieser unterschiedlichen Interviewpartner und ihres individuellen Zugangs zur Zielgruppe der Untersuchungsgegenstand sehr gut herausgearbeitet werden konnte.

Für die Auswertung der erhobenen Daten wurde die Methode der qualitativen Inhaltsanalyse nach Mayring ausgewählt. „Die Stärke der qualitativen Inhaltsanalyse liegt in ihrem systematischen, regelgeleiteten Vorgehen“ (Mayring 2010, S. 124). Zentrales Instrument ist dabei das in einem induktiven Verfahren entwickelte Kategoriensystem (vgl. Mayring 2010, S. 49ff.).

\section{Gestaltungsmittel nachhaltiger medienpädagogischer Bildungs- angebote für Senioren}

Die anhand der inhaltlichen Strukturanalyse herausgefilterten Informationen bieten einen breiten Einblick in Voraussetzungen, Rahmenbedingungen sowie Gestaltungsmöglichkeiten innovativer Bildungsangebote für die Zielgruppe Senioren. Mit Absicht wird im Folgenden der Begriff ,Bildungsangebote anstelle des Begriffs ,Projekt ${ }^{`}$ verwendet, da die Ergebnisse der Untersuchung teilweise stark vom Projektbegriff abweichen. Zudem wird erkenntlich, dass das abgeschlossene Konzept eines Projekts nicht unbedingt den Ansprüchen der Lernbedürfnisse älterer Menschen entspricht. Die Betreuung im Nachhinein, ob offen oder regelmäßig, bildet einen wesentlichen Teil der Gestaltung einer nachhaltigen Lernatmosphäre für Ältere. Hierbei entsteht ein Widerspruch zu den Aussagen von Baacke, der Medienvermittlung grundsätzlich als Projektarbeit versteht (vgl. Baacke 1999c, S. 86). Dieser Aspekt zeichnet sich als Besonderheit der medienpädagogischen Arbeit mit Älteren ab. Offene Bildungsangebote gewähren darüber hinaus die Möglichkeit, nach einer längeren Phase des eigenständigen Austestens, Nachfragen zu stellen. Die Vermittlungsperson kann in diesen Fällen häufig in den Hintergrund treten, da sich die Senioren gegenseitig weiterhelfen.

In der Analyse der Interviews wurde das praxisgeleitete Verständnis von nachhaltigem Lernen der wissenschaftlichen Definition nach Schüßler (vgl. Schüßler 2007) gegenübergestellt. Es zeigte sich, dass im Selbstverständnis der Interviewpartner bereits mehrere Dimensionen nachhaltigen Lernens enthalten waren. Sie sprachen unterschiedliche Aspekte an, die vorrangig der graduellen und der Strukturdimension zugeordnet werden konnten.

Für die Befragten äußerte sich die strukturelle Wissenssubdimension beispielsweise im unabhängigen Weiterarbeiten der Älteren, nachdem das Projekt beendet war. Auch Verhaltensänderungen wurden als nachhaltige Wirkung anerkannt, selbst wenn diese eine bewusste Ablehnung des Bildungsgegenstandes bedeutete. Die Ausbildung von Kompetenzen, welche Handeln auch in anderen Kontexten ermöglichen, stand jedoch nicht im praktischen Vordergrund. In der theoretischen Definition nimmt dies hingegen einen höheren Stellenwert ein.

Die Breite der graduellen Dimension zeichnete sich für die Befragten in der persönlichen Fortbildung und auch in der Weitergabe erlangten Wissens aus. Zudem ließen sich aus der Analyse noch weitere Aspekte zur Beförderung nachhaltiger Lernwirkungen erkennen. Dabei spielte die Gruppenzusammensetzung, ob altershomogen oder -heterogen keine große Rolle. Dennoch sollte diese Entscheidung nicht willkürlich getroffen werden (vgl. de Groote/ Fricke 2010, S. 49). Bei handlungsorientierten Bildungsansätzen erwiesen sich Kleingruppen als geeigneter, um eine angemessene Betreuung sicherstellen zu können.

Die Eingrenzung des Informationsgehalts auf die relevanten Anwendungsfelder wurde ebenso wie eine einfache, verständliche Vermittlungssprache und der Professionalisierungsgrad der Vermittlungsperson betont (vgl. de Groote/ Fricke 2010, S. 45). Diese sollte sich darüber hinaus an den Bedürfnissen der Teilnehmenden orientieren, um flexi- 
bel darauf eingehen zu können. Hierzu erweist es sich als notwendig, diese genau zu kennen und ihre Lernbedürfnisse analysieren zu können. Dieser Aspekt deckt sich mit der strukturellen Dimension nachhaltigen Lernens (vgl. Schüßler 2007, S. 208). Kleinschrittiges Vorgehen „und nicht zu viel auf einmal zu erklären" (de Groote/ Fricke 2010, S. 51) zeichnet sich im Bereich der Medienbildung von Senioren ebenfalls als wesentliches Gestaltungsmittel ab. Dies konnten die praxiserfahrenen Interviewpartner bestätigen.

Ein besonderer Aspekt, der den Bereich der Seniorenbildung von der Pädagogik abgrenzt, ist die Motivation, mit der Ältere an Angeboten teilnehmen. Sie lernen freiwillig und zu persönlichen Zwecken. „Ältere denken praktisch. Lernen hat in der nachberuflichen Phase nur dann einen Wert, wenn es eine Beziehung zum eigenen Leben hat" (de Groote/ Fricke 2010, S. 46). Je häufiger ältere Menschen zudem positive Erfahrungen mit neuen Medien in ihrem Alltag machen, desto eher öffnen sie sich für diese Themen und entsprechende Bildungsangebote. „Häufig werden Bildungsangebote nämlich deshalb abgewehrt, verdrängt, ignoriert oder das Wissen gerät in Vergessenheit, weil die Angebote nicht als Ressource genutzt werden konnten, um die eigene Handlungsfähigkeit zu sichern." (Schüßler 2007, S. 210)

Bei der Konzeption medienpädagogischer Angebote für ältere Menschen gilt es außerdem, einen weiteren wichtigen Aspekt zu beachten. Erwachsene und Senioren lernen auf andere Weise und mit anderen Methoden als Kinder und Jugendliche. Der Prozess der Aneignung ist ,gekennzeichnet durch Spezifika wie die Biografie, die Erfahrung mit Medien und die Weise, in der Medien in das eigene Leben eingebaut wurden" (Schorb 2011, S. 74).

\section{Entwicklung der ,Mediengera- gogik‘ als neue Aufgabe}

Die Auswertung der explorativen Untersuchung zeigt, dass es zahlreiche Gestaltungsmittel und Bedingungen gibt, welche die Nachhaltigkeit medienpädagogischer Bildungsarbeit mit Senioren befördern können und welche sich von der Arbeit mit Jüngeren unterscheiden. Dieser Bereich könnte in Zukunft, in Abgrenzung zur Medienpädagogik, unter dem Begriff der ,Mediengeragogik“ gefasst werden. Dieser hat sich im wissenschaftlichen Kontext bisher noch nicht etablieren können und bezeichnet ein wichtiges neues Praxis- und Forschungsfeld. Der Begriff leitet sich ab aus der Geragogik, welche sich vorrangig mit der „Gestaltung von Lernund Bildungsprozessen im Alter" (Bubolz-
Lutz 2010, S. 11) befasst und der Medien $p \ddot{a}$ dagogik, also der Medienbildung von Kindern und Jugendlichen. Die Aufgabe der Geragogik ist es, „Vorbehalte der unterschiedlichen Nutzergruppen Älterer zu ermitteln und dann entsprechende Bildungsansätze zu entwickeln“ (Bubolz-Lutz 2010, S. 193). Der Begriff ,Mediengeragogik‘ bezeichnet folglich die Medienbildung Älterer. „Die geragogische Praxis verfolgt das Ziel, durch professionell angeregte und begleitete Lernprozesse Ältere dabei zu unterstützen, ihre individuellen Ressourcen und Potenziale zu erkennen und weiterzuentwickeln“ (Bubolz-Lutz 2010, S. 12; Herv. i. O.). Aus der Literatur geht eindeutig hervor, dass Medienbildung nicht mit Erreichen der Volljährigkeit oder mit dem Schulabschluss beendet sein darf (vgl. von Hippel 2011, S. 688): „In einer Informationsgesellschaft, in der die Gruppe der Alten zunehmend an wirtschaftlicher, politischer und gesellschaftlicher Macht gewinnt, erscheint die Frage nach der Förderung von Medienkompetenz der Älteren essentiell“" (Bogen u.a. 2005, S. 10). Somit zeichnet sich eine Notwendigkeit der Ausbildung des Mediengeragogik-Begriffs ab.

In der weiteren Entwicklung sollten die notwendigen Gestaltungsmittel der mediengeragogischen Praxis um die Perspektive der Teilnehmer erweitert werden. Zudem wurden in dieser Forschung ausschließlich Projekte ausgewertet und damit nur auf einen Bruchteil des Bildungsangebots eingegangen. Da sich dauerhafte, regelmäßige Betreuungsangebote als besonders geeignet für nachhaltige Lernprozesse gezeigt haben, sollten diese insbesondere Beachtung in der Ausarbeitung des Begriffs finden.

Zudem werden ,angesichts einer zunehmend höheren Qualifikation der Teilnehmer [...] neue, anspruchsvollere Angebote zu entwickeln sein, die zunehmend stärker individuellen Bildungsbiografien gerecht werden müssen" (Bundesministerium für Familie, Senioren, Frauen und Jugend 2005, S. 151). Das Angebot wird sich in den nächsten Jahren also weg von der reinen Vermittlung von Faktenwissen und hin zu innovativen gestalterischen Bildungsangeboten entwickeln müssen. Ältere Menschen auf den aktuellen Stand der Technik zu bringen, wird bei den jungen Alten nicht mehr notwendig sein.

Problematisch zeigt sich jedoch der Zugang eher bildungsferner Älterer sowie in ihrer Mobilität eingeschränkter Senioren. „Insgesamt zeigen die Befunde, dass Personen mit höherer Schul- und Berufsausbildung überproportional an Bildungsangeboten partizipieren, sodass Bildungsungleichheiten im Alter eher verstärkt werden“ (Bundesministerium für Familie, Senio- 
ren, Frauen und Jugend 2005, S. 148). Hier müssen Mittel und Wege gefunden werden, Bildungsangebote für diese Zielgruppe zu etablieren. Die Möglichkeiten mobiler Endgeräte und des mobilen Internets erleichtern diese Aufgabe zusehends.

Medienpädagogische Angebote für Senioren zielen häufig darauf ab, Praxishilfen zu bieten und den Alltag durch die Nutzung neuer Medien zu erleichtern und auszugestalten. Aus diesem Grund ist es von besonderer Relevanz, Nachhaltigkeit als Zielkategorie des Bildungsangebots zu definieren. In dieser Arbeit wurden einige Anregungen zur Gestaltung mediengeragogischer Angebote zusammengestellt, welche durch tiefergehende und flächendeckendere Befunde noch erweitert werden können. Sie sollen dazu anregen, bei der Konzeption mediengeragogischer Angebote Überlegungen zur bewussten und nachhaltigen Gestaltung miteinzubeziehen.

\section{Anmerkungen}

1 Dieser Text geht auf meine gleichnamige Bachelorarbeit zurück, die ich 2014 im Bachelor-Studiengang Kultur- und Medienbildung an der PH Ludwigsburg erstellte. Die Arbeit wurde von Prof. Dr. Horst Niesyto (Abteilung Medienpädagogik) und Damaris Nübel (Abteilung Kultur- und Medienbildung) betreut.

2 Im Folgenden wird auf eine begriffliche Differenzierung zwischen der maskulinen und der femininen Form verzichtet. Dies soll keine Benachteiligung oder Diskriminierung von Bevölkerungsgruppen darstellen, sondern dient lediglich der Vereinfachung und der erhöhten Lesbarkeit.

\section{Literatur}

ARD/ZDF-Onlinestudie (2013): ard-zdfonlinestudie.de. URL: http://www.ard-zdfonlinestudie.de, Abruf: 14.09.2014

Baacke, Dieter (1999): Projekte als Formen der Medienarbeit. In: Baacke, Dieter u.a. (Hrsg.): Handbuch Medien: Medienkompetenz. Modelle und Projekte, Bonn, S. 31-35.

Berner, Frank/ Tesch-Römer, Clemens/ Wurm, Susanne (2013): Altersbilder im Wandel. In: Aus Politik und Zeitgeschichte (APuZ), Jg. 63, 2013, Heft 4-5, S. 3-8.

BITCOM e.V. (2013): Soziale Netzwerke 2013. Dritte, erweiterte Studie. Eine repräsentative Untersuchung zur Nutzung sozialer Netzwerke im Internet. URL: http:// www.bitkom.org/files/documents/ SozialeNetzwerke_2013.pdf, Stand: 21.10.2013, Abruf: 08.03.2014
Bogen, Cornelia u. a. (2005): Alte Menschen und Medien. Alter im Spannungsfeld zwischen Kultur und Medien. In: Bogen, Cornelia/ Domaschke, Madlen/ Pabst, Sabine (Hrsg.): Alte Menschen und Medien. Alter im Spannungsfeld zwischen Kultur und Medien, Halle, S. 1-18.

Bubolz-Lutz, Elisabeth u.a. (2010): Geragogik. Bildung und Lernen im Prozess des Alterns. Das Lehrbuch, Stuttgart.

Bundesministerium für Familie, Senioren, Frauen und Jugend (2005): Fünfter Bericht zur Lage der älteren Generation in der Bundesrepublik Deutschland. Potenziale des Alters in Wirtschaft und Gesellschaft. Der Beitrag älterer Menschen zum Zusammenhalt der Generationen. URL: http:// www.bmfsfj.de/RedaktionBMFSFJ/ A b teilung 3/Pdf-Anlagen/ fuenfteraltenbericht,property= pdf,bereich $=$ bmfsfj, sprache $=$ de, $r w b=$ true.pd f, Stand: 08.2005, Abruf: 09.03.2014

De Groote, Kim/ Nebauer, Flavia (2008): Kulturelle Bildung im Alter. Eine Bestandsaufnahme kultureller Bildungsangebote für Ältere in Deutschland. München.

De Groote, Kim/ Fricke, Almuth (2010): Kulturkompetenz 50+. Praxiswissen für die Kulturarbeit mit Älteren. München.

Fischer, Daniel/ Nemnich, Claudia (2013): Bildung für nachhaltige Entwicklung innovativ gestalten. In: Overwien, Bernd/ Rode, Horst (Hrsg.): Bildung für nachhaltige Entwicklung. Lebenslanges Lernen, Kompetenz und gesellschaftliche Teilhabe. Opladen, S. $169-183$.

Gudjons, Herbert (2008): Handlungsorientiert lehren und lernen. Schüleraktivierung. Selbsttätigkeit. Projektarbeit. Bad Heilbrunn.

Hartung, Anja/ Reißmann, Wolfgang/ Schorb, Bernd (2005): Perspektiven für eine Medienkompetenzförderung im höheren Lebensalter. In: Bogen, Cornelia/ Domaschke, Madlen/ Pabst, Sabine (Hrsg.): Alte Menschen und Medien. Alter im Spannungsfeld zwischen Kultur und Medien, Halle, S. 119135.

Kolland, Franz (2008): Lernbedürfnisse, Lernarrangements und Effekte des Lernens im Alter. In: Aner, Kirsten/ Karl, Ute (Hrsg.): Lebensalter und Soziale Arbeit. Ältere und alte Menschen. Baltmannsweiler, S. 174-186.

Ladner, Michael (2013): Nachhaltiges Lernen. Gestaltungsnotwendigkeiten kognitiver Prozesse im Rahmen des nachhaltigen Lernens. München.

Mayring, Philipp (2010): Qualitative Inhaltsanalyse. Grundlagen und Techniken. 11., 
aktualisierte und überarbeitete Auflage, Weinheim und Basel.

Netzwerk für Senioren-Internet-Initiativen Baden-Württemberg (o.J.): Veranstaltungsreihe SIHmobil. URL: http://www.netzwerk-sii -bw.de/startseite.html, Abruf: 22.04.2014

Pfäffli, Brigitta K. (2005): Lehren an Hochschulen. Eine Hochschuldidaktik für den Aufbau von Wissen und Kompetenzen. Bern.

Reichert-Garschhammer, Eva u.a. (2013): Projektarbeit im Fokus. Fachliche Standards und Praxisbeispiele für Kitas, Berlin.

Schorb, Bernd (2011): Erfahren und neugierig. Medienkompetenz und höheres Lebensalter. In: Kupser, Thomas/ Pöttinger, Ute (Hrsg.): Mediale Brücken. Generationen im Dialog durch aktive Medienarbeit. München, S. 49-79.

Schüßler, Ingeborg (2007): Nachhaltigkeit in der Weiterbildung. Theoretische und empirische Untersuchungen zum nachhaltigen Lernen. Baltmannsweiler.

Statistisches Bundesamt (2009): Bevölkerung Deutschlands bis 2060. 12. Koordinierte Bevölkerungsvorausberechnung. URL: https: / / w w w.destatis.de/DE/ Publikationen/Thematisch/Bevoelkerung/ VorausberechnungBevoelkerung/ BevoelkerungDeutschland2060Press e 512242040999004 . p d f ? _blob=publicationFile, Stand: 18.11.2009, Abruf: 09.03.2014

Stiftung Digitale Chancen (o.J.): Tablet-PCs für Seniorinnen und Senioren. URL: http:// www.digitale-chancen.de/tabletpcs / index.cfm/secid.33, Abruf: 22.04.2014

Volkshochschulverband Baden-Württemberg (o.J.): Mobile Wege ins Internet. URL: h t t p : / / w w w . v h s - b w . d e / smartphone4bestager/index.html, Abruf: 22.04.2014

von Hippel, Aiga (2011): Erwachsenenbildung und Medien. In: Tippelt, Rudolf/ von Hippel, Aiga (Hrsg.): Handbuch Erwachsenenbildung/Weiterbildung. 5. Auflage, Wiesbaden, S. 687-706.

ZAWiW (2013): Silberwissen. URL: http:// www.uni-ulm.de/einrichtungen/zawiw/ projektbereiche/neue-medieninternet/ silberwissen.html, Stand: 16.12.2013, Abruf: 22.04.2014 